\title{
Erythrodermic psoriasis and palmoplantar hyperkeratosis successfully treated with secukinumab: a case report
}

\author{
Martino Carriero \\ Dermatology Unit, Poliambulatorio Grottaglie, Grottaglie - Italy
}

\begin{abstract}
Introduction: Erythrodermic psoriasis (EP) is a rare and severe form of psoriasis that affects $1 \%$ to $2.25 \%$ of patients, increasing mortality risk. To date, very few therapies have been approved for the treatment of this condition. Recently, biological therapies that specifically target inflammatory cytokines have improved the management and treatment of EP. Secukinumab, a human monoclonal antibody that specifically targets interleukin-17A (IL-17A), has been shown to be beneficial in different psoriasis settings.

Methods: We report the case of a 72-year-old man affected by persistent EP and severe palmoplantar hyperkeratosis whose condition was not resolved after two rounds of treatment with prednisone and therapy with cyclosporine.

Results and conclusions: Treatment with secukinumab significantly improved the symptoms of palmoplantar hyperkeratosis as early as the first week, with a decrease of psoriasis area and severity index (PASI) score from 60 to 10 , showing almost complete remission after 1 month. Consistent with the current literature, secukinumab treatment showed promising and encouraging clinical outcomes in the treatment of the patient's EP. However, more studies are needed to clarify the IL-17-dependent mechanism in the pathophysiology of EP.
\end{abstract}

Keywords: Biologic therapy, Erythrodermic psoriasis, Interleukin-17, Monoclonal antibody, Palmoplantar hyperkeratosis, Secukinumab

\section{Introduction}

Psoriasis is an immune-mediated inflammatory disease with unknown etiology, characterized by the presence of papules and plaques of various morphology and severity over the surface of skin (1). Psoriasis is classified as an inflammatory autoimmune disease in which an excessively aberrant hyperproliferation of keratinocytes is present, along with dilated, hyperplastic blood vessels and inflammatory infiltration of leukocytes predominantly into dermis (2). These features typically present as red skin patches that are itchy and scaly. As with other autoimmune diseases, psoriasis may have a systemic outcome beyond the skin, also affecting the

Received: November 4, 2021

Accepted: February 23, 2022

Published online: March 7, 2022

\section{Corresponding author:}

Martino Carriero

Dermatology Unit

Poliambulatorio Grottaglie

Via Tiziano 26

Grottaglie (TA), 74023 - Italy

dott.martino.carriero@alice.it joints, nails, and other organs (2). Currently, there is no cure available, and psoriasis imposes a substantial negative impact on the quality of life (QoL) of patients. Psoriasis can occur at any age and is most common in the age group 50-69 years. The reported country-specific prevalence of psoriasis ranges between $0.09 \%$ and $11.4 \%$, making psoriasis a serious global problem (1), with approximately $3 \%$ of the US population and around 125 million people affected worldwide, ranging from $0.5 \%$ in Asian regions to $8 \%$ of the population in Norway. Male and female populations are equally affected in most regions (3).

Psoriasis may occur in different forms, such as plaque psoriasis (characterized by dry scaly patches), which represents $80 \%-90 \%$ of psoriasis cases; pustular psoriasis (contains puslike fluid mainly infiltrated with white blood cells); erythrodermic psoriasis (EP, characterized by exfoliation of fine scaly skin with pain and itching); guttate psoriasis (characterized by drop-like dots); and inverse psoriasis (affects the flexure surfaces and characterized by smooth inflamed lesions) (1).

The pathogenesis of psoriasis is not completely understood, and the exact mechanism remains elusive, although the literature suggests that different genetic, epigenetic, and environmental factors may be responsible for the onset of psoriasis (2). Although not inherited in a Mendelian fashion, a familial predisposition may be present, which significantly 
increases the relative risk of psoriasis in families with a positive history among first-degree and second-degree relatives of patients, compared to the general population (2). Epigenetic factors including dysregulated deoxyribonucleic acid (DNA) methylation levels, abnormal histone modification, and microribonucleic acid (miRNA) expressions have been recognized as crucial players in the pathophysiology of psoriasis. Among other factors, excessive activation of the adaptive immune system and the interplay of immune cells and cytokines are thought to play a role in the onset of psoriasis $(2,3)$. An initial secretion of cytokines that activate myeloid dendritic cells, from plasmacytoid dendritic cells, keratinocytes, natural killer T cells, and macrophages, is thought to initiate the first steps of the pathogenesis of psoriasis (3).

\section{Erythrodermic psoriasis}

Among the other forms of psoriasis, EP represents a rare and severe form that affects $1 \%$ to $2.25 \%$ of patients with psoriasis. The clinical characteristics of this form of psoriasis are the presence of erythematous, edematous, often exfoliative lesions that affect over $75 \%$ of the body surface area (4), and are often associated with numerous systemic symptoms such as fever, tachycardia, lymphadenopathy, arthralgia, and fatigue (5). There is a substantial risk of mortality due to transepidermal fluid and nutrient loss, and which in severe cases may lead to multiorgan failure and death (4).

EP generally develops in patients with poorly controlled psoriasis and as a result of the abrupt withdrawal of systemic medications such as corticosteroids, drug reactions to medications such as lithium, and underlying systemic infections (5). For a clear and correct diagnosis of EP, two general clinical subtypes that define the disease must be considered. The first subtype is characterized by psoriatic plaques that gradually differentiate and develop into generalized erythroderma, although, overall, the plaques remain differentiable from the erythroderma. This form of EP has a stable course and a good prognosis. In contrast, the second EP subtype is more common in a psoriatic arthritis setting and plaques usually cannot be distinguishable from whole-body erythema (6). As with psoriasis, the exact pathogenesis of EP is not fully clarified. The current literature suggests the involvement of an unbalanced T-helper (Th)1/Th2 differentiation in favor of the Th2 phenotype and its related cytokine secretion. Interleukin (IL)-4 and IL-13, in particular, have been shown to be elevated in EP relative to both psoriasis patients and healthy controls. Several studies have demonstrated that anti-tumor necrosis factor (TNF)- $\alpha$ agents improve EP outcomes, suggesting that TNF- $\alpha$ may play a role in the pathogenesis of EP (6).

Although difficult and challenging, the management of EP is possible through several treatment options. The US National Psoriasis Foundation had suggested, in 2010 in their consensus guidelines, the use of cyclosporine or infliximab as first-line therapy in unstable cases, with acitretin and methotrexate reserved for more stable cases. Since then, other therapies and treatment strategies have emerged, including topical treatment with steroids and vitamin D analogues, as well as phototherapy. Other studies have also demonstrated the efficacy of systemic agents, including second-generation retinoids (acitretin) and immunosuppressive drugs (methotrexate and cyclosporine), which showed complete remission and significant improvement in EP outcomes.

Recently, another class of drugs, called biological agents, has demonstrated high efficacy in EP management. Biological therapies represent an emerging class of immunosuppressive drugs, which, thanks to their enhanced selectivity to specific cytokines, may represent a valid alternative to the canonical treatments. Biological therapies include TNF- $\alpha$ inhibitors, IL-12/23 inhibitors, and IL-17 inhibitors (6).

\section{Secukinumab: drug description and focus on the treatment of EP}

As mentioned, an unbalanced proinflammatory response and massive cytokine secretion may promote and sustain worsening symptoms of EP. Besides Th1 and Th2, the recent discovery of the new class of T-helper cells, Th17, also highlighted the possible role of the proinflammatory cytokine IL-17 (secreted by Th17) in the pathogenesis of EP (7). Some environmental triggers, such as physical trauma, drugs, or infections, release proinflammatory cytokines, including IL-23 and TNF- $\alpha$. Differentiation of T helper into Th17 cells and the release of cytokines, such as IL-17, promote keratinocyte proliferation, which, in the setting of EP, also release additional ILs and chemokines (8). The homodimeric glycoprotein IL-17A belongs to the IL-17 family and through its receptor complex IL-17RA/IL-17RC binds to keratinocytes, dendritic cells, dermal fibroblast, and endothelial cells.

Under physiological conditions, normal levels of IL-17A promote, upon binding, the proliferation of the keratinocytes necessary for healing, and protect against infectious agents. However, in psoriatic patients, levels of IL-17A are elevated, and correlate with the severity of the disease. Keratinocytes themselves, when stimulated, also synthesize many cytokines that can induce epidermal hyperplasia (autocrine growth factors) or neoangiogenesis (paracrine growth factors), resulting in worsening of EP and initiating a reverberating loop that perpetuates pro-proliferative and proinflammatory stimuli (9). Consistently, blocking IL-17A results in an improvement of psoriasis lesions, suggesting a key role in the pathogenesis of EP (8). Currently, one anti-IL-17A biological agent is approved for the treatment of plaque psoriasis, secukinumab, a monoclonal antibody that targets IL-17A (10).

Secukinumab is a novel biologic agent that specifically targets IL-17A. It is a fully human monoclonal antibody, and many clinical trials have demonstrated its efficacy in the treatment of plaque psoriasis, psoriatic arthritis, and ankylosing spondylitis (AS) (11-14). Indications approved by the European Medicines Agency (EMA) include adult and pediatric psoriasis, psoriatic arthritis, axial spondyloarthritis (axSpA), AS and non-radiographic axial spondyloarthritis (nr-axSpA) (15). The rationale behind the efficacy and mechanism of the action of secukinumab is a targeted approach to block the disease process at a very early stage along with a safe adverse event profile (16). By targeting IL-17A, secukinumab thereby blocks its binding with IL-17R and consequent expression of cytokines. Blocking the IL-17A pathway leads to a normalization of the inflammatory processes and thus combats epidermal 

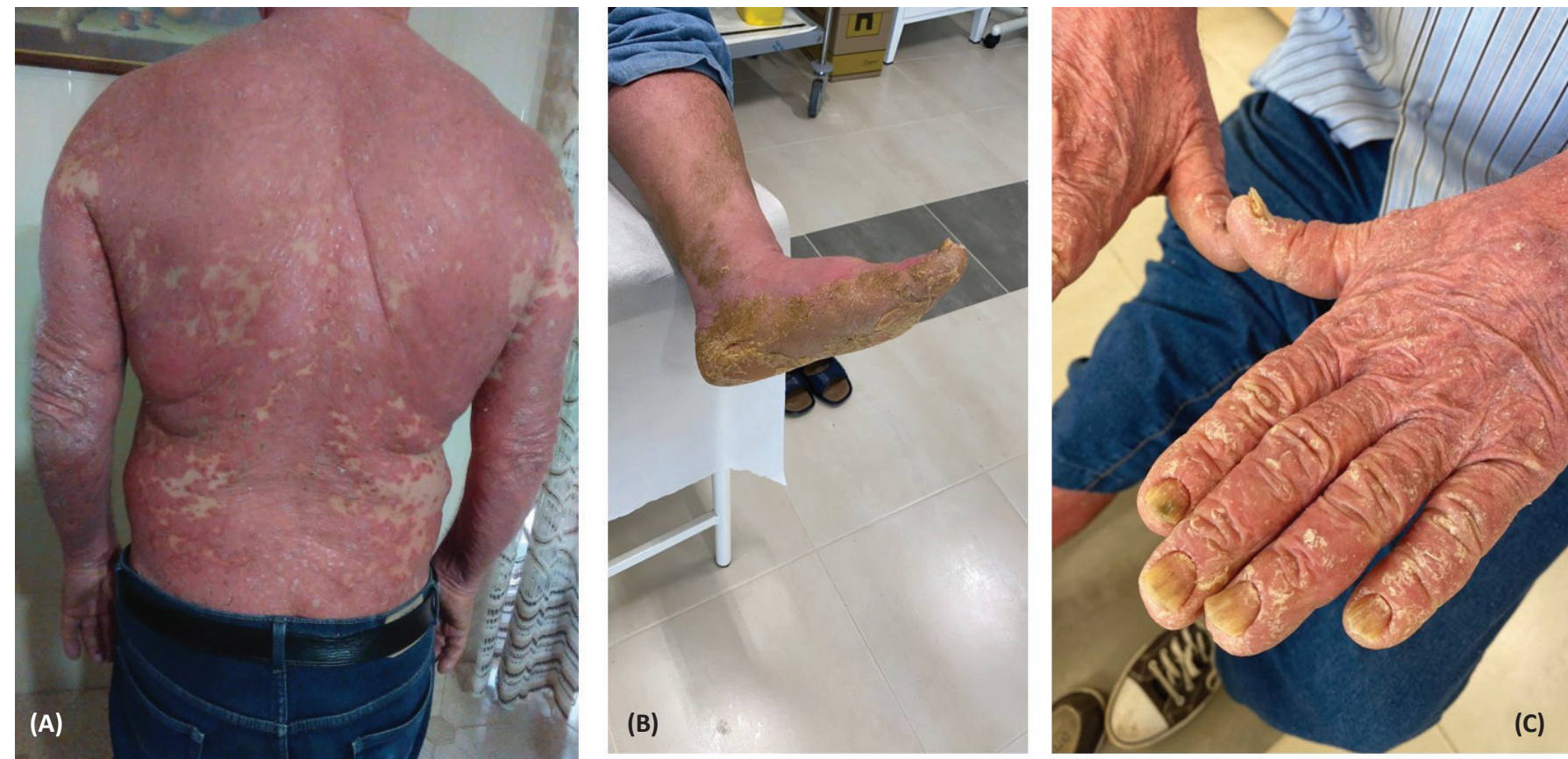

Fig. 1 - Widespread erythrodermic psoriasis (A) and palmoplantar hyperkeratosis $(B, C)$ at initial presentation before secukinumab treatment.

hyperproliferation, T-cell infiltration, and excessive expression of pathogenic genes (16).

Several clinical trials have demonstrated the efficacy of secukinumab in the treatment of psoriasis (11-14). Two phase III, double-blind, 52-week trials (ERASURE and FIXTURE) (17) evaluated the efficacy of secukinumab in patients with moderate-to-severe plaque psoriasis. The ERASURE study enrolled 738 patients, while FIXTURE study included 1,306 patients. In both studies patients received either placebo or secukinumab subcutaneously once a week for 5 weeks, but in the FIXTURE study patients also received etanercept (50 mg twice a week for 12 weeks, then once a week). Results from the ERASURE study showed that a psoriasis area and severity index (PASI) 75 score at week 12 was achieved by $81.6 \%$ and $71.6 \%$ of patients administered with $300 \mathrm{mg}$ and $150 \mathrm{mg}$ of secukinumab, respectively, and by $4.5 \%$ of placebo recipients. Corresponding results from FIXTURE showed that $77.1 \%$ of patients administered $300 \mathrm{mg}$ of secukinumab, $67 \%$ of those administered $150 \mathrm{mg}$ of secukinumab, $44 \%$ of etanercept recipients, and only $4.9 \%$ of placebo patients achieved PASI 75 score at week 12 (17).

Another multicenter, international, retrospective, pilot study enrolled 13 EP patients who were treated with a loading dose of $300 \mathrm{mg}$ of subcutaneous secukinumab at weeks $0,1,2,3$, and 4, followed by $300 \mathrm{mg}$ every 4 weeks (18). Results from this study showed that the response rate to secukinumab was $10 / 13$ patients $(77 \%)$, with a median time to clearance of 3 weeks (1.5-3 weeks). No recurrences were registered in the 52-week follow-up and a PASI score of 90 was achieved by $10 / 13$ patients. These results demonstrated that secukinumab remains a valid and effective therapeutic option for EP (18).

\section{Case presentation}

In this real-life clinical experience of the treatment of psoriasis with secukinumab drug, we report the case of a 72-year-old man, in general good health condition, who contacted the dermatologic outpatient clinic of the author on March 5, 2020, during the pandemic lockdown. The patient reported widespread EP with severe palmoplantar hyperkeratosis (shown in Fig. $1 \mathrm{~A}-\mathrm{C}$ ), and he was not taking any other drugs or medications. He was started on prednisone $(25 \mathrm{mg}$ one tablet) treatment for 8 days and at half-dose for 8 more days. In addition, topical therapy with mometasone furoate cream was prescribed. On March 16, 2020, blood chemical tests were in the normal range, and the patient continued therapy with cyclosporine (100 mg twice a day) until July 2020. The patient's erythroderma significantly improved, but his palmoplantar hyperkeratosis persisted. Due to onset of hypertensive seizures and increased creatininemia, the patient discontinued the treatment. Then, after a pause of a few weeks, the patient resumed prednisone treatment in August 2020, due to the persistence of severe hyperkeratosis. However, there was no response or improvement.

On September 1, 2020, the patient started secukinumab treatment at the dose of $300 \mathrm{mg}$ administered by two subcutaneous injections of $150 \mathrm{mg}$ each (15); significant signs of clinical resolution were observed during the induction phase, for both erythrodermic psoriasis and palmoplantar 

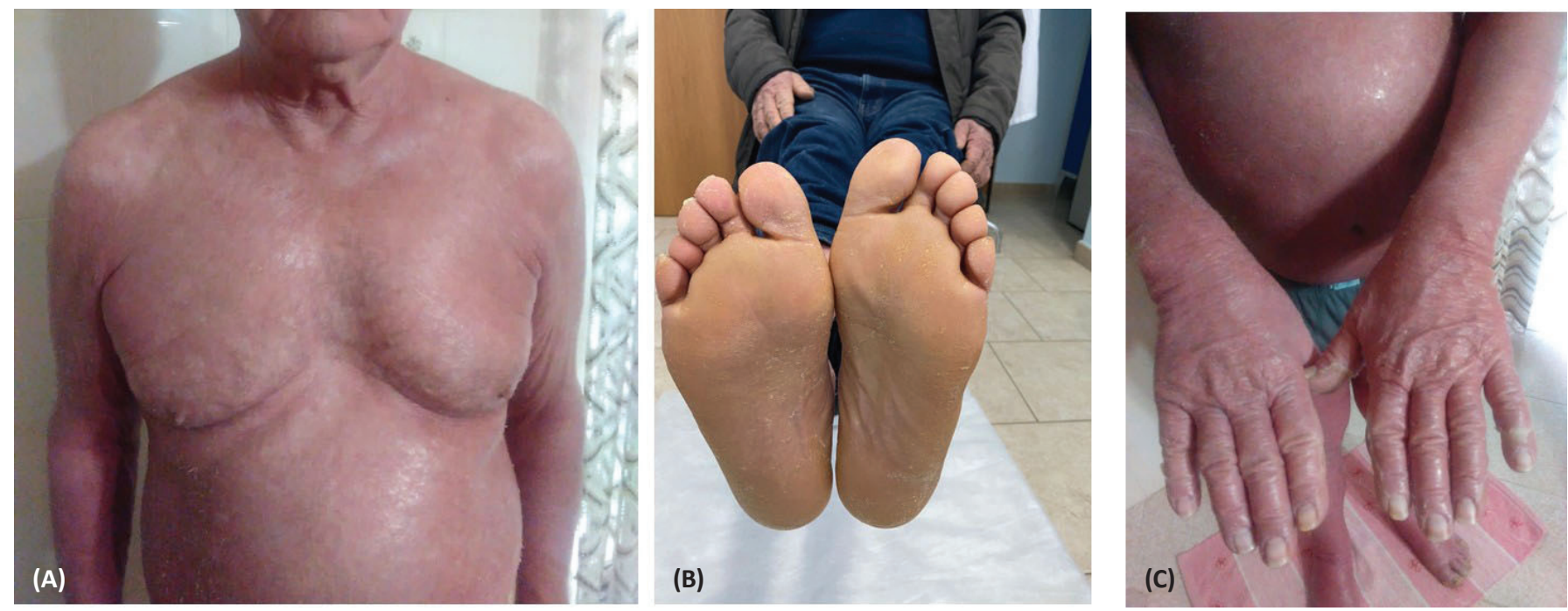

Fig. 2 - Improved erythrodermic (A) and palmoplantar hyperkeratosis (B, C) after secukinumab treatment.

hyperkeratosis. The patient is currently under maintenance treatment with secukinumab and his general condition is good. The PASI score at the first visit was 60, and decreased to 20 after induction (shown in Fig. 2A-C). At a follow-up visit, conducted at the end of February 2021, the patient showed an almost complete remission, with a PASI score of 10 . As of January 2022 the patient, in perfect health conditions with normal hematochemical tests, presents a complete remission of the clinical picture.

\section{Discussion and conclusions}

Although the treatment options for EP have greatly expanded in recent years, psoriasis and its severe forms remain a major public health concern. Treatment of EP with secukinumab has shown promising and encouraging clinical outcomes, and the present case report showed data consistent with other case reports from the literature. Weng et al (19) reported a series of 10 cases in which secukinumab was prescribed for EP. Data from those case reports showed that at week $16,4 / 10$ patients and $7 / 10$ patients, respectively, were able to achieve PASI 90 and PASI 75 scores (19). In this very first and unique case for our clinical practice, the patient, affected by palmoplantar psoriasis, but who also presented a very severe form of EP, showed significant signs of clinical resolution during the induction phase, with few side effects. The initial PASI score of the patient was 60 , which significantly decreased to 20 after the induction and to 10 after the follow-up visit at 6 months, suggesting complete remission of EP. However, although treatment outcomes with secukinumab are promising, additional controlled trials with extended follow-up are needed to better understand the link between IL-17 inhibition and the resolution of EP.

\section{Acknowledgments}

The author thanks Enrica Piras, PhD, who provided editorial assistance and Ray Hill, an independent medical writer, who provided English-language editing prior to submission on behalf of Health Publishing \& Services Srl. Financial support for medical editorial assistance was provided by Novartis Farma, Italy. The funder was not involved in the conception, drafting and writing of this article or in the decision to submit it for publication.

\section{Author contributions}

Dr. Carriero: conception and design of the work, acquisition and analysis of data, draft of the work; agreed to be accountable for all aspects of the work in ensuring that questions related to the accuracy or integrity of any part of the work are appropriately investigated and resolved.

\section{Statement of ethics}

Written informed consent for publication (including images) has been obtained from the patient. The research was conducted ethically in accordance with the Declaration of Helsinki.

\section{Disclosure}

Conflict of interest statement: The author has no conflicts of interest to declare.

Funding sources: This research received no specific grant from any funding agency in the public, commercial, or not-for-profit sectors. Data availability statement: All data generated or analyzed during this study are included in this article. Further enquiries can be directed to the corresponding author.

\section{References}

1. Rajguru JP, Maya D, Kumar D, Suri P, Bhardwaj S, Patel ND. Update on psoriasis: a review. J Family Med Prim Care. 2020; 9(1):20-24. CrossRef PubMed

2. Deng $Y, C h a n g C, L u$. The inflammatory response in psoriasis: a comprehensive review. Clin Rev Allergy Immunol. 2016; 50(3):377-389. CrossRef PubMed 
3. Armstrong AW, Read C. Pathophysiology, clinical presentation, and treatment of psoriasis: a review. JAMA. 2020;323(19):19451960. CrossRef PubMed

4. Reynolds KA, Pithadia DJ, Lee EB, Liao W, Wu JJ. A systematic review of treatment strategies for erythrodermic psoriasis. J Dermatolog Treat. 2021;32(1):49-55. CrossRef PubMed

5. Carrasquillo OY, Pabón-Cartagena G, Falto-Aizpurua LA, et al. Treatment of erythrodermic psoriasis with biologics: a systematic review. J Am Acad Dermatol. 2020;83(1):151-158. CrossRef PubMed

6. Singh RK, Lee KM, Ucmak D, et al. Erythrodermic psoriasis: pathophysiology and current treatment perspectives. Psoriasis (Auckl). 2016;6:93-104. CrossRef PubMed

7. Fitch E, Harper E, Skorcheva I, Kurtz SE, Blauvelt A. Pathophysiology of psoriasis: recent advances on IL-23 and Th17 cytokines. Curr Rheumatol Rep. 2007;9(6):461-467. CrossRef PubMed

8. Berg SH, Balogh EA, Ghamrawi RI, Feldman SR. A review of secukinumab in psoriasis treatment. Immunotherapy. 2021; 13(3):201-216. CrossRef PubMed

9. Chiricozzi A, Krueger JG. IL-17 targeted therapies for psoriasis. Expert Opin Investig Drugs. 2013;22(8):993-1005. CrossRef PubMed

10. Wasilewska A, Winiarska M, Olszewska M, Rudnicka L. Interleukin-17 inhibitors. A new era in treatment of psoriasis and other skin diseases. Postepy Dermatol Alergol. 2016;33(4):247-252. CrossRef PubMed

11. Reich K, Warren RB, Coates LC, Di Comite G. Long-term efficacy and safety of secukinumab in the treatment of the multiple manifestations of psoriatic disease. J Eur Acad Dermatol Venereol. 2020;34(6):1161-1173. CrossRef PubMed
12. Deodhar A, Mease PJ, McInnes IB, et al. Long-term safety of secukinumab in patients with moderate-to-severe plaque psoriasis, psoriatic arthritis, and ankylosing spondylitis: integrated pooled clinical trial and post-marketing surveillance data. Arthritis Res Ther. 2019;21(1):111. CrossRef

13. Baraliakos X, Gossec L, Pournara E, et al. Secukinumab in patients with psoriatic arthritis and axial manifestations: results from the double-blind, randomised, phase $3 \mathrm{MAXI}$ MISE trial. Ann Rheum Dis. 2021;80(5):582-590. CrossRef PubMed

14. Kiltz U, Sfikakis PP, Gaffney K, et al. Secukinumab use in patients with moderate to severe psoriasis, psoriatic arthritis and ankylosing spondylitis in real-world setting in Europe: baseline data from SERENA Study. Adv Ther. 2020;37(6):2865-2883. CrossRef PubMed

15. Cosentx-Summary of Product Characteristics. Available at: Online (Accessed October 2021).

16. Frieder J, Kivelevitch D, Menter A. Secukinumab: a review of the anti-IL-17A biologic for the treatment of psoriasis. Ther Adv Chronic Dis. 2018;9(1):5-21. CrossRef PubMed

17. Langley RG, Elewski BE, Lebwohl M, et al; ERASURE Study Group; FIXTURE Study Group. Secukinumab in plaque psoriasisresults of two phase 3 trials. N Engl J Med. 2014;371(4):326338. CrossRef PubMed

18. Damiani G, Pacifico A, Russo F, et al. Use of secukinumab in a cohort of erythrodermic psoriatic patients: a pilot study. J Clin Med. 2019;8(6):770. CrossRef PubMed

19. Weng HJ, Wang TS, Tsai TF. Clinical experience of secukinumab in the treatment of erythrodermic psoriasis: a case series. $\mathrm{Br} \mathrm{J}$ Dermatol. 2018;178(6):1439-1440. CrossRef PubMed 